References:

Nam-Chul Ha, Sang-Taek Oh, Jae Young Sung, Kyeung Ah Cha, Mann Hyung Lee \& Byung-Ha Oh "Supramolecular assembly and acid resistance of Helicobacter pylori urease" (2001) Nature Structural Biology 8, 505-509.

Strugatsky, D., McNulty, R.M., Munson, K., Chen, C.-K., Soltis, S.M., Sachs, G., Luecke H. "Structure of the proton-gated urea channel from the gastric pathogen Helicobacter pylori”" (2013) Nature 493, 255-258.

Luecke, H. \& Sachs, G. "Helicobacter pylori's Achilles' Heel" (2013) Immuno-Gastroenterology 2, 76.

Keywords: Helicobacter pylori, Urease, Cryo-EM
MS10- Hydrogen-bonding \& weak interactions studied by neutrons and X-rays

Chairs: Prof. Marta E. G. Mosquera, Dr. Matthew Blakeley

MS10-P01

\section{Frequency and hydrogen bonding geometry of nucleobase homodimers in small molecule crystals}

Malgorzata Cabaj ${ }^{1}$, Paulina Dominiak ${ }^{1}$

1. Biological and Chemical Research Centre, Department of Chemistry, University of Warsaw, Warsaw, Poland

email: mcabaj@chem.uw.edu.pl

The issue of various nucleobase pairs and their interactions occurring in RNA and DNA has been already studied from many different perspectives. In this survey, we wanted to approach the subject from more "chemical" point of view and see how nucleobases interact inside crystals. Our study is based on geometric data like bond lengths and bond angles taken from the Cambridge Structural Database (CSD), as well as types of protonation of investigated nucleobase, if the hydrogen positions were available.

We searched for nucleobase homodimers resembling those found in RNA and DNA, which means two nucleobases interacting through at least two hydrogen bonds formed in the molecule plane. The investigated compound were derivatives of adenine, guanine, hypoxanthine, thymine, uracil, and cytosine. We divided our findings into many categories including types of dimers, their protonation and if the N9 or N1 (for purines and pyrimidines respectively) is substituted with only hydrogen or larger substituent that may influence the way the nucleobase forms dimers. In our study, we analyze the various dependencies between the geometry of the molecule and what types of structures it prefers to form. We investigated not only neutral forms of nucleobases, but protonated too, and analyzed how protonation and charge influence the ability of a molecule to form homodimers.

Our study finds that for purines the most active edge is Hoogsten edge, taking part in the formation of dimers found in more than half of the investigated structures. Mixed interactions between the Hoogsteen edge and Watson-Crick edge are also very common, as they allow the molecules to align into infinite ribbons.

For pyrimidines, the situation is much different, as the various possible interactions between Watson-Crick edges of molecules dominate the charts. Only uracil behaves differently from thymine and cytosine, as it eagerly forms trans Watson-Crick - Hoogsten interaction, namely the Calcutta dimer. For all nucleobases, there is a common trend of sugar edge interactions being more frequent if the molecule is substituted with hydrogen in N9 or N1. 
Fig. 1. Examples of homodimers of adenine and cytosine, with the naming of the edges

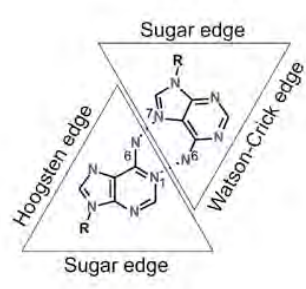

Adenine

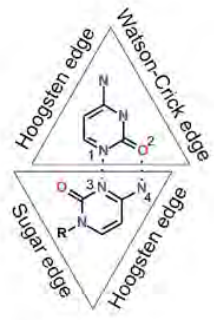

Cytosine
Keywords: nucleobases, hydrogen bonding

\section{VS10-P02}

\section{Analysis of the hydrogen bonds in the crystal structures of the pyrrole-2-yl- dichloromethyl ketone derivatives}

\author{
Małgorzata Domagała $^{1}$, Alina T. Dubis ${ }^{1}$, Marcin Palusiak ${ }^{1}$ \\ 1. Faculty of Chemistry, University of Lodz, Lodz, Poland \\ email: mdomagala@uni.lodz.pl
}

Pyrroles are both important natural products and crucial starting materials for the synthesis of porphyrins. Nevertheless, a brief survey of the Cambridge Structural Database [1] shows that there are almost 500 instances of the organic structures with a 2-substituted pyrrole core and almost 100 instances with the pyrrol-2-yl carbonyl core. What is more, only a few crystal structures of aromatic N-unsubstituted monopyrroles have been reported. In crystal structures of investigated compounds, we expected the existence of the net of hydrogen and halogen bonding as it was in case of the trichloromethyl derivative of pyrroloketone [2]. However, $\mathrm{X}$-ray structure determinations showed only the latter one. In an extension of ongoing studies on the hydrogen bonding patterns in porphyrins and dipyrromethanes, we were also interested in the strengths and types of hydrogen bonds of isolated pyrrole units. For initial studies we choose 2,2-dichloro-1-(1H-pyrrol-2-yl) ethanone and 2,2-dichloro-1-(1-methyl-1H-pyrrol-2-yl) ethanone. The calculations have been performed using Gaussian03 sets of codes, and by means of the density functional methods (DFT). The H-bonding characteristics of the complexes have been investigated with the use of Bader's theory QTAIM [3].

References:

[1] Allen, F. H. (2002). Acta Cryst. B58, 380-388.

[2] Bilewicz, E. et al. (2007). J. Mol. Struct. 829, 208-211.

[3] Bader, R. F. W. (1990). Atoms in Molecules: A Quantum Theory; Oxford University Press, New York.

Keywords: hydrogen bond, monopyrolle 